\title{
Translating XML Update Language into SQL
}

\author{
Pensri Amornsinlaphachai*, Nick Rossiter and M. Akhtar Ali \\ School of Computing, Engineering \& Information Sciences, Northumbria University, Newcastle upon Tyne, UK
}

\begin{abstract}
Several techniques for translating XML query languages into SQL have been proposed, but no work to date translates XML update languages into SQL since XQuery has not provided any update statements. However, there is a suggestion from $\mathrm{W} 3 \mathrm{C}$ indicating that an update version of XQuery will be proposed in the near future. Furthermore, one major advantage of updating XML documents via a relational database is that the preservation of constraints can be transferred to the database engine; thus our main contributions are translating the XML update language, extending XQuery into SQL and translating recursive updates into PL/SQL. XQuery is a functional language whereas $\mathrm{SQL}$ is a declarative language; therefore, translation cannot be performed directly, so several techniques such as rewriting rules and graph mapping are used in our work.
\end{abstract}

Keywords: XML update language, SQL, XQuery, translation, recursive function.

\section{Introduction}

The emergence of XML as an effective standard for representation of (semi-)structured data on the Web has motivated a host of researches in the area related to XML such as storing $[6,15,22]$, querying $[24,25,3]$ and updating $[13,19,31]$ XML documents. In the area of querying XML documents, several query languages such as Lorel [1], XPath [32], XMLQL [5], XQL [23] and XQuery [33] have been proposed while several translation techniques have been presented for translating these languages into SQL. For example, [28] translates Lorel, [16] translates XPath, [12, 35] translate XML-QL, $[29,10]$ translate XQL and $[9,7,27]$ translate XQuery. In the area of updating XML documents, several researchers pay attention to designing XML update languages such as XUpdate [34], SiXDML [26], XML-RL Update Language [19] and XML Update Extension [30], but none of the published work has proposed translating these languages into SQL.

Our motivation comes from three reasons as follows. Firstly, none of the published work has presented the translation of XML update language into SQL although many researchers have proposed a number of XML update languages. Secondly, translating recursive querying in XQuery is still an open problem. However, in our research we focus on translating the update language. Thus, instead of translating recursive querying, we translate recursive updating in the XML update language, an extension to XQuery. Nevertheless, it is still possible to apply our technique to translate recursive querying in XQuery. Finally, if updating is performed directly on an XML document in the manner of the native XML database, much work must be handled such as preserving constraints, while updating XML documents via a relational database has the advantage of using the database engine to preserve constraints. This is because before updating XML documents, both structure and constraints of XML will be mapped to the schema of the relational database, while the XML update language will be translated into SQL and this SQL is used to update data in the database.

In this paper, we will express how to translate the XML update language into SQL, including translating a recursive function into $\mathrm{PL} / \mathrm{SQL}$ since XQuery has not provided statements for

\footnotetext{
${ }^{*}$ This work was supported by the Royal Thai Government via Nakhonratchasima Rajabhat University.
} 
querying data recursively. For this task, the recursive function is needed. In order to demonstrate our translation techniques, a database representing XML documents and an update language are necessary. In this respect we adapt existing researches to our work because our main contribution is elsewhere.

The rest of this paper is organized as follows. Related work is discussed in section 2. A database and a language for updating are described in section 3. Section 4 presents techniques for translating XML update language into SQL commands and section 5 presents how to translate the recursive function into PL/SQL. Finally, conclusion and further work are discussed in section 6 .

\section{Related Work}

Several techniques $[8,11,20]$ for translating XML query languages have been proposed and these techniques may be classified according to the method for representing XML in the database. There are three methods for representing XML to the database: by the edge approach, by the shredding approach and as a view created from the database.

There are several ways in which XML query languages are translated into SQL; however, we will describe only the general approach of translating XPath into SOL based on representing XML by the edge and shredding approaches since XPath is used as a part of other XML query languages. Until now, for translation based on representing XML as view, we see only the translation of XQuery into SQL. Because XQuery is a functional language whereas SQL is a declarative language, translation cannot be performed straightforwardly: there is no general approach for translating it and thus we will describe each technique for translating XQuery that we have found.

The general approach $[35,14]$ for translating XPath into SQL based on representing XML by the edge approach is as follows. In storing XML by the edge approach, elements and paths of elements are kept in one table and attributes and path of attributes are kept in another table. Thus, to translate XPath to SQL, PathIDs from
XPath are created and then an SQL statement is used to retrieve rows in tables, based on the condition that the PathIDs derived from XPath are the same as the path-IDs kept in the tables. The path-IDs are used to join the tables.

The general approach [16] for translating XPath into SQL by the shredding approach is as follows. In storing XML by the shredding approach, complex elements are converted to tables while simple elements and attributes are converted to fields; thus, to translate XPath into SQL, relations and fields can be identified from their path expressions and the relations are then joined together.

In the case of translation based on representing $\mathrm{XML}$ as a view created from the database, Fernandez M. et al. [8] translate XQuery to SQL by using a view forest. Semantically, a view forest defines a mapping from a relational database to an XML document. Any XQuery expression can be rewritten as a forest view. The SQL fragments are stored in each node of the view forest. The internal nodes will contain FROM and WHERE clauses, whereas the leaf nodes contain only SELECT clause. Another translation method proposed by Fernandez M. et al. [7] is translating XQuery into SQL by decomposing an XML view definition into smaller SQL queries and submitting the decomposed SQL queries to the database.

Shanmugasundaram J. et al. [27] translate XQuery into SQL as follows. Firstly, the XQuery query is parsed and converted to an internal query representation called XML Query Graph Model (XQGM). Secondly, the query is composed with XML views to which it refers. Finally, optimizations are performed to eliminate the construction of intermediate XML fragments and predicates are pushed down.

To summarize, none of the previous work translates XML update languages into SQL although several XML update languages, which are extensions to XQuery, have been proposed and the official update version of XQuery may be presented shortly; moreover, fully fledged relational technology can be exploited when XML is updated via the relational database. The varying methods for translating the XML query language into SQL are summarized in Table 1. 


\begin{tabular}{|c|c|c|c|c|c|}
\hline Researches & $\begin{array}{l}\text { Linear } \\
\text { Recursion }\end{array}$ & $\begin{array}{l}\text { Non-linear } \\
\text { Recursion }\end{array}$ & Optimisation & $\begin{array}{l}\text { Represent } \\
\text { XML to } \\
\text { Database } \\
\end{array}$ & $\begin{array}{l}\text { Translated } \\
\text { Language }\end{array}$ \\
\hline Krishnamurthy R. et al. [16] & $\mathrm{Y}$ & Y & $\mathrm{N}$ & Shredding & $\begin{array}{l}\text { Path } \\
\text { Expression }\end{array}$ \\
\hline Fong J.; Dillon T. [10] & $\mathrm{N}$ & $\mathrm{N}$ & $\mathrm{N}$ & Shredding & XQL \\
\hline Jain S. et al. [11] & $\mathrm{N}$ & $\mathrm{N}$ & $\mathrm{Y}$ & Shredding & XSLT \\
\hline Shanmugasundaram J. et al. [28] & $\mathrm{Y}$ & $\mathrm{N}$ & $\mathrm{N}$ & Shredding & Lorel \\
\hline Fernandez M. et al. [7] & $\mathrm{N}$ & $\mathrm{N}$ & $\mathrm{Y}$ & XML view & XQuery \\
\hline Fernandez M. et al. [8] & $\mathrm{N}$ & $\mathrm{N}$ & $\mathrm{Y}$ & XML view & XQuery \\
\hline Shanmugasundaram J. et al. [27] & $\mathrm{N}$ & $\mathrm{N}$ & $\mathrm{Y}$ & $\mathrm{XML}$ view & XQuery \\
\hline Shimura T. et al. [29] & $\mathrm{N}$ & $\mathrm{N}$ & $\mathrm{N}$ & Edge approach & XQL \\
\hline Jensen, E.C. et al. [12] & $\mathrm{N}$ & $\mathrm{N}$ & $\mathrm{N}$ & Edge approach & XML-QL \\
\hline Manolescu I. et al. [20] & $\mathrm{N}$ & $\mathrm{N}$ & $\mathrm{N}$ & Edge approach & Quilt \\
\hline Manolescu I. et al. [9] & $\mathrm{N}$ & $\mathrm{N}$ & $\mathrm{N}$ & Edge approach & XQuery \\
\hline Khan L. et al. [14] & $\mathrm{N}$ & $\mathrm{N}$ & $\mathrm{N}$ & Edge approach & XPath \\
\hline Yoshikawa M. [35] & $\mathrm{N}$ & $\mathrm{N}$ & $\mathrm{N}$ & Edge approach & XPath \\
\hline
\end{tabular}

Table 1. Comparison of Techniques for Translating XML Query Languages into SQL.

\section{A Database and a Language for Updating}

To demonstrate how to translate the XML update language into SQL, a database and an update language are necessary. In this section, we apply and adapt existing work so that we can express our translation techniques in the next section. In this section firstly, we will describe representing XML in a relational database and secondly, we will present an XML update language used for updating.

\subsection{Mapping XML to Relational Database}

To map XML to a relational database, we follow the technique presented in work [16] since it is compact and easy to understand. The researchers of this work represent mapping via annotations on the DTD schema graph; however, we adjust the rules in the part for mapping the recursive form and naming key fields. The DTD schema graph is shown in Figure 1.
The annotations on the graph correspond to the following decomposition. Each non-leaf node is mapped to a table name and each leaf node is mapped to a column name. Each table has an id as the primary key, while each table which is not the root has parent-id as a foreign key for preserving document structure. The name of the primary key is the table-name followed by 'id' while the name of a foreign key is the same as the name of the primary key of the parenttable. When an element has an attribute whose type is ID, this ID will be used as the primary key. For IDREF(s) and the recursive structure, a separate table is created to hold the primary keys of tables of the referencing element and the referenced element. The name of the separate table is the name of the referencing element followed by the name of the referenced element. The database derived from mapping the graph shown in Figure 1 is given in Figure 2 in the form of a database schema graph. In the graph, the symbol (T) stands for table while the arrow with a dashed-line stands for recursion. 


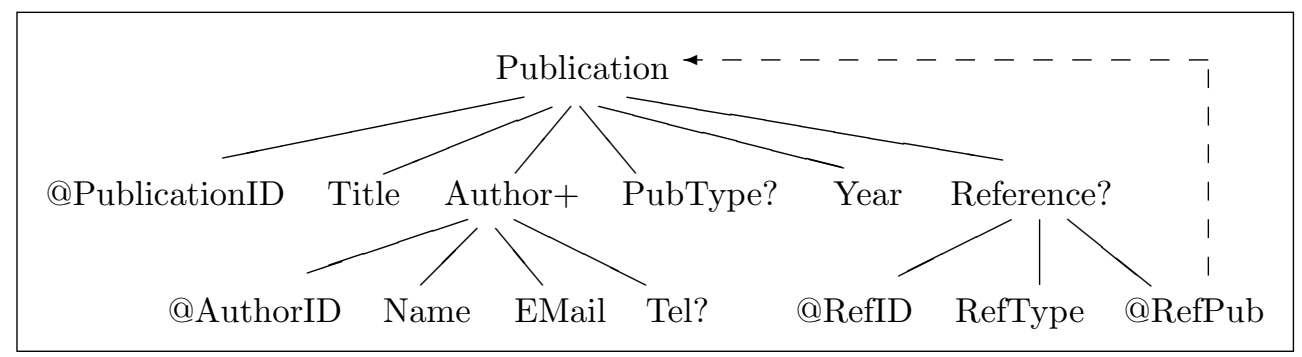

Fig. 1. DTD schema graph.

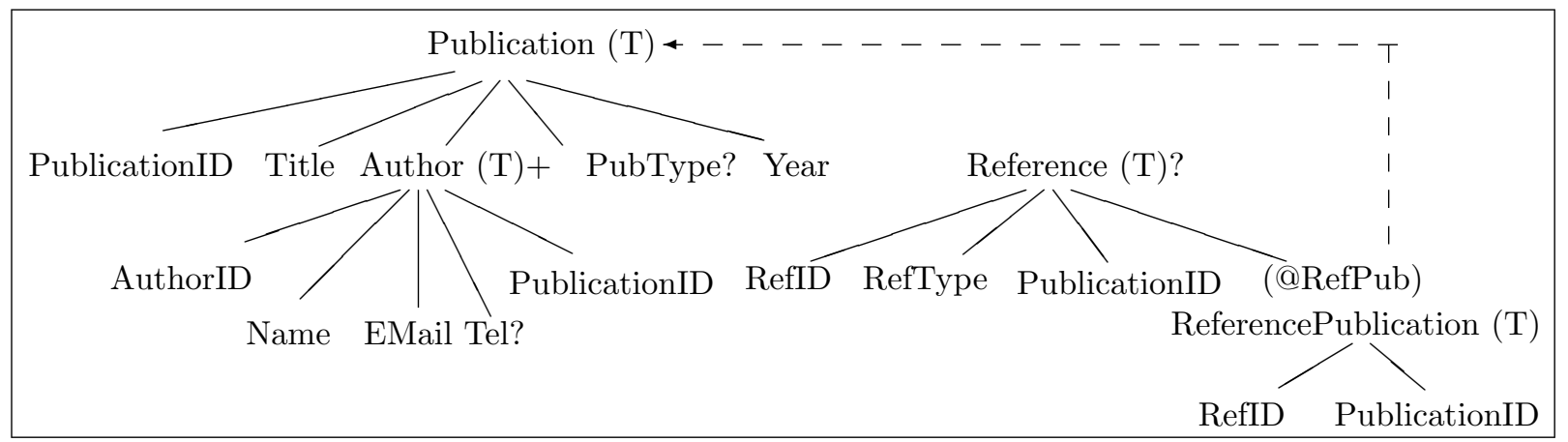

Fig. 2. Database schema graph.

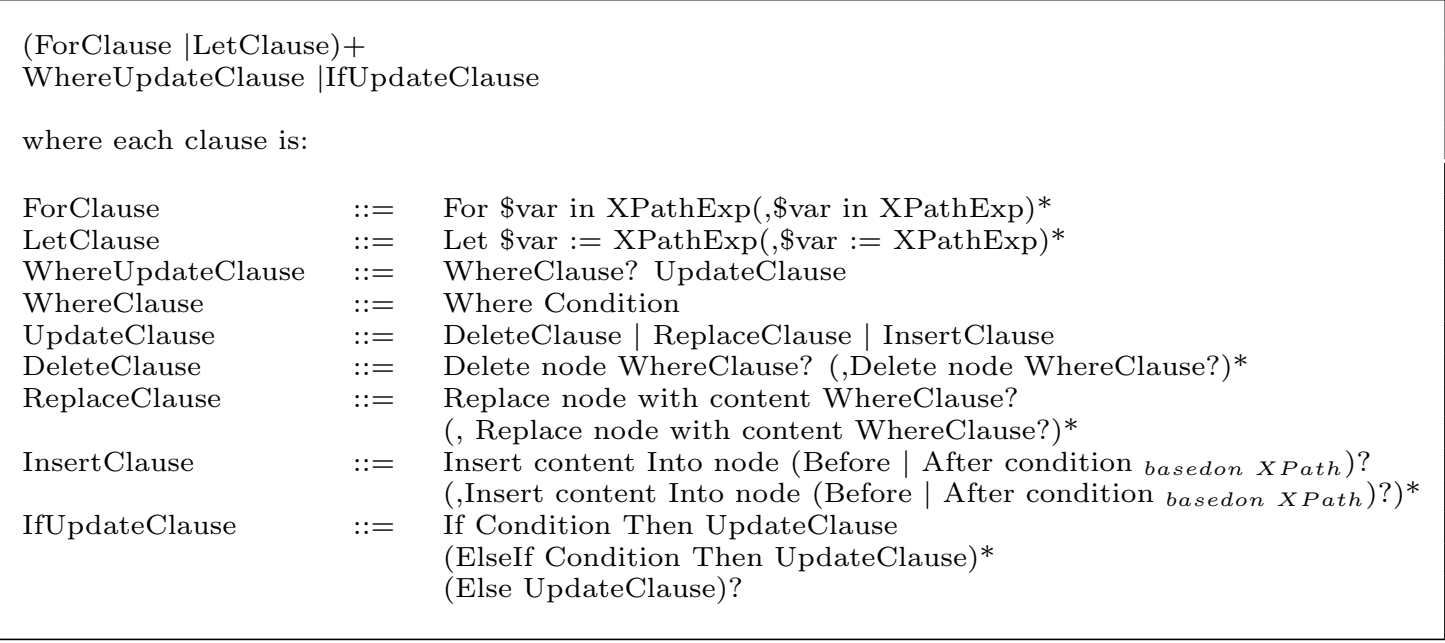

Fig. 3. Syntax of XML Update Language.

For propagating the constraints of XML to the relational database, the rules proposed in work $[18,17,31]$ can be applied. By applying these rules, preserving the constraints of XML is pushed to the database engine.

\subsection{An XML Update Language}

For the update language, we adapt the syntax proposed by Tatarinov, I. et al. [30] and the syntax of XQuery [33]. The syntax after this adaptation is shown in Figure 3. The semantics of the update language is the same as that presented in [30].

\section{Translating XML Update Language into SQL}

When compared with existing XML query languages, XQuery is the most powerful, providing many features $[21,4,33]$. In this section, five important features will be translated 
into SQL: FLW(R|I|D), an abbreviation for a For-Let-Where-(Replace|Insert|Delete) expression, conditional expression, quantifier, aggregate functions and (non-recursive) user-defined function. XQuery is a functional language whereas SQL is a declarative language; therefore translating the XML update language, an extension to XQuery, into SQL is not straightforward; thereby several techniques such as rewriting rules will be used during the translation. In this section, firstly, four techniques for translating the update language will be described. Secondly, the steps for translating the update language into SQL will be proposed and finally an example will be presented.

\subsection{Four Techniques for Translating XML Update Language}

Our translation uses four main techniques: update/delete join commands (joins in update/delete commands), rewriting rules, graph mapping and optimization rules for translating XML update language into SQL. These four techniques are as follows.

\subsubsection{Update/Delete Join Commands}

In the SQL standard, update/delete join commands cannot be performed; however the translation of XML update commands can produce joins of several tables. Therefore, it is necessary that we translate XML update commands into update/delete join commands and then rewrite them to SQL with sub-query commands. The syntax of update/delete join commands is as follows:

- Syntax of joins in update command

Update table whose fields will be updated from all related tables

Set field $1=$ value 1, field $2=$ value $2, \ldots$

Where Condition;

- Syntax of joins in delete command

Delete table whose data will be deleted from all related tables

Where Condition;

Note: Insert ... select-joins can already be performed in the SQL standard.

\subsubsection{Rewriting Rules}

There are six categories of rewriting rules: FLW $(\mathrm{R}|\mathrm{I}| \mathrm{D})$ expression, aggregate function, quantifier, conditional expression, (non-recursive) user-defined function and SQL rewriting rules. The first five categories are classified according to features of the update language and these features will be rewritten as SQL functions while the last category of rewriting rules: SQL rewriting rules is used to rewrite update/delete join commands as SQL commands. In this section, we explain the SQL function and then describe the six categories of rewriting rules.

SQL Functions. To translate XML update commands, all clauses of XML update commands must be rewritten as SQL functions. SQL functions are conceptual functions representing the operations of SQL commands. The SQL functions are used to group XML update clauses and their conditions together since one XML update command can consist of several update clauses and each update clause can have its own condition. Thus, these update clauses are grouped by using function number (funcNo) which is a parameter of every SQL function. The funcNo 0 will be assigned to ForClause, LetClause and WhereClause of the XML update command. These clauses will be shared clauses for the UpdateClause. Each update clause will have its own funcNo, a running number starting from 1 . The update clause and its own condition will have the same funcNo. The SQL functions are as follows:

1. bindF (path, $\$$ var, funcNo)

2. bindL (path, \$var, funcNo)

3. insert (node, value funcNo, funcNo)

4. delete(node, funcNo)

5. update(node, value |funcNo, funcNo)

6. where|LogicalOper(node, ComparisonOper, value (funcNo, funcNo)

7. aggFunc(node, funcNo) where aggFunc $:=\max |\min |$ count |avg sum

8. group_by(node, funcNo)

9. having(aggFunc(node), ComparisonOper, value (funcNo, funcNo)

10. $\operatorname{select}$ (node, funcNo) 
Four SQL functions, where|Logical(), having(), insert() and update( ), have the parameter value funcNo since sometimes the value in the predicate, in inserting or in updating, is not the constant value, but it may come from selecting a value from other nodes. Hence in this case, funcNo has the same number as that for the funcNo of the select() function.

Rewriting rules for $\operatorname{FLW}(\mathbf{R}|\mathbf{I}| \mathbf{D})$. The expression FLW $(\mathrm{R}|\mathrm{I}| \mathrm{D})$ will be rewritten as SQL functions as follows:

1. For \$var in XPathExp is rewritten as: bindF(XPathExp, \$var, funcNo)

2. Let $\$$ var $:=$ XPathExp is rewritten as: bindL(XPathExp,\$var, funcNo)

3. Where predicate is rewritten as: where (node, ComparisonOper, value (funcNo, funcNo)

4. LogicalOper predicate is rewritten as: LogicalOper(node, ComparisonOper, value (funcNo, funcNo)

5. For $\$$ var in $\mathrm{XPathExp}$ predicate is translated into:

For \$var in XPathExp Where predicate Then this clause is rewritten as SQL functions according to rules $1,3,4$.

6. Let $\$ \mathrm{var}:=\mathrm{XPathExp} \mathrm{Predicate}_{\text {is }}$ is translated into:

Let \$var := XPathExp Where predicate Then this clause is rewritten as SQL functions according to rules 2-4.

7. Select node |Return node is rewritten as: select (node, funcNo)

8. Replace node with content is rewritten as: update(node, content's value, funcNo)

9. Delete node delete(node, funcNo)

is rewritten as:

10. Insert simple content into node is rewritten as:

Insert(node, content's value, funcNo)

11. Insert complex content Into node

The complex content is shredded into many simple contents. The Insert command is rewritten in the form of the commands based on the simple contents which are in turn rewritten as SQL functions as follows:
Define: complex content $\mathrm{e}_{c}=\left\{\mathrm{e}_{1}, \mathrm{e}_{2}, \ldots\right.$, $\left.\mathrm{e}_{i-1}, \mathrm{e}_{i}, \mathrm{a}_{1}, \mathrm{a}_{2}, \ldots \mathrm{a}_{i}\right\}$ where $\mathrm{e}_{1}, \mathrm{e}_{2}, \ldots, \mathrm{e}_{i-1}$, $\mathrm{e}_{i}$ are elements, $\mathrm{a}_{1}, \mathrm{a}_{2}, \ldots, \mathrm{a}_{i}$ are attributes, $\mathrm{e}_{i}=\left\{\mathrm{e}_{i 1}\left\{\mathrm{e}_{i 2} . .\left\{\mathrm{e}_{i i}\right\} ..\right\}, \mathrm{a}_{i 1}, \mathrm{a}_{i 2}, \ldots, \mathrm{a}_{i i}\right\}$.

$\mathrm{v}_{e 1}, \mathrm{v}_{e 2}, \ldots, \mathrm{v}_{e i-1}, \mathrm{v}_{a 1}, \mathrm{v}_{a 2}, \ldots, \mathrm{v}_{a i}$ are values of $\mathrm{e}_{1}, \mathrm{e}_{2}, \ldots, \mathrm{e}_{i-1}, \mathrm{a}_{1}, \mathrm{a}_{2}, . ., \mathrm{a}_{i}$ and $\mathrm{v}_{e i i}, \mathrm{v}_{a i 1}$, $\mathrm{v}_{a i 2}, \ldots, \mathrm{v}_{a i i}$ are values of $\mathrm{e}_{i i}, \mathrm{a}_{i 1}, \mathrm{a}_{i 2}, \ldots, \mathrm{a}_{i i}$ respectively.

Insert $\mathrm{e}_{c}$ Into node is rewritten as: insert(node $/ \mathrm{e}_{c}$, , funcNo) insert (node $/ \mathrm{e}_{c} / \mathrm{e}_{1}, \mathrm{v}_{e 1}$, funcNo) $\operatorname{insert}\left(\operatorname{node} / \mathrm{e}_{c} / \mathrm{e}_{2}, \mathrm{v}_{e 2}\right.$, funcNo)

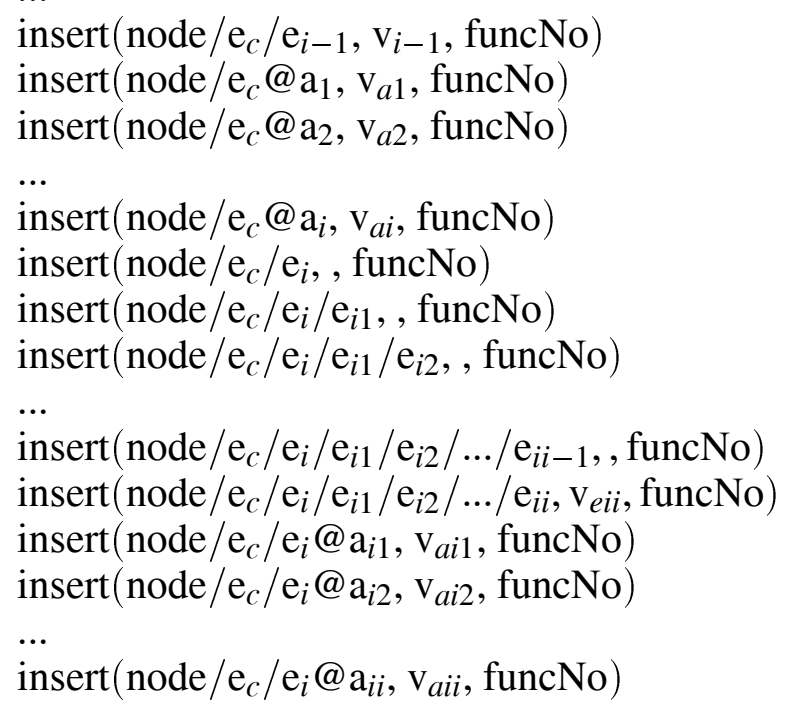

\section{Rewriting rules for aggregate functions.}

1. Define: For \$var1 in XPathExp1 Let \$var2 := \$var1/XPathExp2

Then: aggFunc(\$var2) is rewritten as: aggFunc (\$var2, funcNo) group_by (\$var1, funcNo)

2. Define: Let $\$$ var $:=X P a t h E x p$

Then: aggFunc(\$var) is rewritten as: aggFunc(\$var, funcNo)

3. Define: For \$var1 in XPathExp1

Then: Let \$var2 := \$var1/XPathExp2

Where aggFunc(\$var2) ComparisonOper value is rewritten as:

group_by (\$var1, funcNo)

having(aggFunc(\$var2),ComparisonOper, value, funcNo) 
Rewriting rules for quantifier. In XQuery, there are two quantifiers: existential quantifier (some) and universal quantifier (every). Both quantifiers can be translated into a count () function since the existential quantifier is used to test whether at least one item in the sequence satisfies the condition while the universal quantifier is used to test whether every item in the sequence satisfies the condition; thus, before rewriting these quantifiers to SQL functions, their meanings will first be translated and then rewritten as SQL functions as follows:

1. For \$var1 in XPathExp1

Where some \$var2 in \$var1/XPathExp2

Satisfies (Condition) is translated into:

For \$var1 in XPathExp1

Let \$var2 := \$var1/XPathExp2

Where count $(\$ \operatorname{var} 2)>0$

And Condition

is rewritten as:

\$var1 = bindF (XPathExp1, funcNo)

\$var2 = bindL $(\$ \operatorname{var} 1 /$ XPathExp2, funcNo)

where (node, ComparisonOperator, value |:funcNo, funcNo)

(LogicalOper(node,ComparisonOperator, value |:funcNo, funcNo))*

group_by (\$var1, funcNo)

having (count $(\$ \operatorname{var} 2),>, 0$, funcNo)

2. For \$var1 in XPathExp1

Where every \$var2 in \$var1/XPathExp2

Satisfies (Condition1)

[And Condition2] is translated into:

For \$var1 in XPathExp1

Let \$var2 := \$var1/XPathExp2

Where Condition 1

[And Condition2]

And count $(\$ \operatorname{var} 2)=$

(For \$var3 in XPathExp1

Let \$var4 := \$var3/XPathExp2

Where \$var3 = \$var1

[And Condition2]

Return count (\$var4)

\$var1 $=$ bindF $($ XPathExp1, funcNo $)$

is rewritten as:

\$var2 = bindL $(\$ \operatorname{var} 1 /$ XPathExp2, funcNo)

where (node,ComparisonOper, value |:funcNo, funcNo)

(LogicalOper (node, ComparisonOper, value |:funcNo, funcNo))*

[and(node,ComparisonOper, value |:funcNo, funcNo)
(LogicalOper(node,ComparisonOper, value |:funcNo, funcNo))*

group_by (\$var1, funcNo)

having $(\operatorname{count}(\$ \operatorname{var} 2),=,: 1$, funcNo$)$

$\$ \operatorname{var} 3=\operatorname{bindF}($ XPathExp1, :1 $)$

\$var4 = bindL (\$var3/XPathExp2,:1)

select (count (\$var4),:1)

where (\$var3, =, \$var1, :1)

[and (node,ComparisonOperator,value, :1)

(logical_operator(node,

ComparisonOperator, value, :1))*

group_by $(\$ \operatorname{var} 3,: 1)$

Besides 'some' and 'every' quantifiers, there are two functions: empty() and exists() which can be rewritten as count () functions. These functions and quantifiers can be used along with 'not'. To summarise, the meaning of these functions and quantifiers can be translated before rewriting as follows:

some is translated into count $>0$

not (some) is translated into count $=0$

every is translated into

count $_{\text {predicate }}=$ count $_{\text {without predicate }}$

not (every) is translated into

count $_{\text {predicate }}<$ count $_{\text {without predicate }}$ and count predicate $>0$

empty is translated into $\quad$ count $=0$

not (empty) is translated into count $>0$

exists is translated into count $>0$

not (exists) is translated into $\quad$ count $=0$

Rewriting rule for conditional expression.

The construction

(ForClause|LetClause)+

If $\left(\right.$ Condition $\left._{1}\right)$ then

UpdateStm $_{1}$

Else If (Condition 2$)$ then

UpdateStm$_{2}$

[

Else [If $\left(\right.$ Condition $\left.\left._{n}\right)\right]$ 


\section{UpdateStm $_{n}$}

is translated into a series of commands as follows:

(ForClause|LetClause)+

Where Condition $_{1}$

UpdateStm $_{1}$

(ForClause|LetClause)+

Where Condition 2

And not( Condition 1 )

UpdateStm$_{2}$
(ForClause|LetClause)+

[Where condition ${ }_{n}$ ]

Where $\mid$ And not $\left(\right.$ condition $\left._{1}\right)$

And not $\left(\right.$ condition $\left._{2}\right)$

And not( condition $\left._{n-1}\right)$

UpdateStm $_{n}$

]

The series of commands are then rewritten as SQL functions according to the category of expressions. The number of commands in the series corresponds to the number of conditions if-then-else.

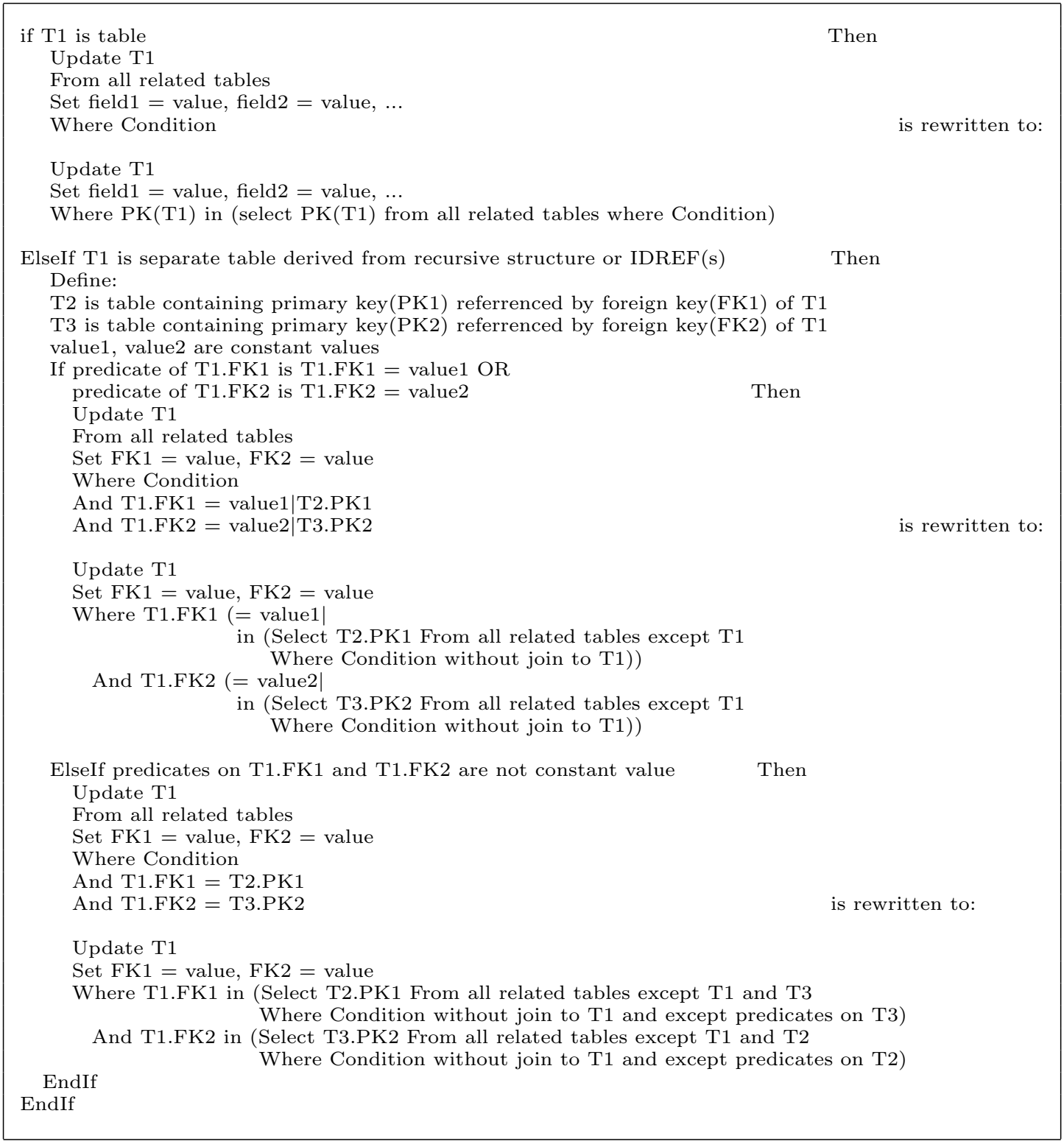

Fig. 4. Rewriting rules for joins in update command. 


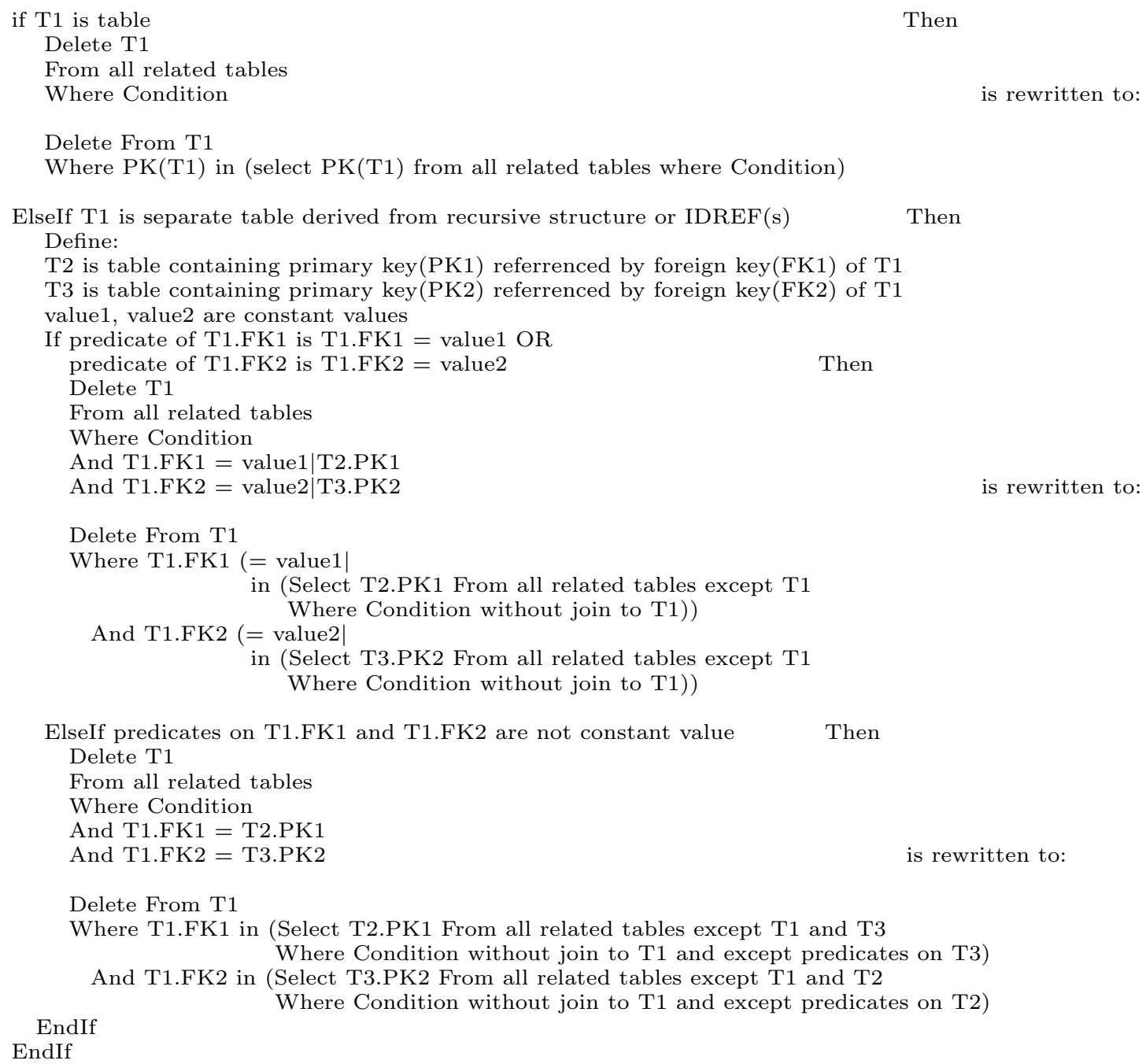

Fig. 5. Rewriting rules for joins in delete command.

\begin{abstract}
Rewriting rules for non-recursive user-defined function. Calls to non-recursive functions are replaced with the body of such functions and parameters are replaced with proper values. After such replacements, the update command is rewritten as SQL functions according to the category of expressions in the command.
\end{abstract}

SQL rewriting rules (rewriting rules for update and delete join commands). These rules are used to rewrite update and delete join commands as SQL commands. Rewriting rules for update join commands are shown in Figure 4 and rewriting rules for delete join commands are shown in Figure 5.

\subsubsection{Graph Mapping}

The purpose of graph mapping is to indicate the SQL functions performed on tables or fields of the database, so that SQL commands can be correctly generated from the graph.

The steps for graph mapping start from creating a graph whose paths correspond to paths in the SQL functions and then the graph is mapped to the database schema graph to identify which node is a table or field. Then the foreign keys for joins tables and join symbols are added to the graph and the SQL functions 
are mapped to the graph. Next pushing the function down to proper nodes of the graph may be performed depending on which function is performed on which node. The graph may then be split into several sub-graphs. The number of sub-graphs corresponds to the number of update operations performed on different tables. Finally, optimization rules are applied to the graph or the sub-graphs and SQL commands or update/delete join commands are generated from the graph or the sub-graphs.

\subsubsection{Optimization Rules}

There are three techniques for optimization as follows:

1. Eliminate unnecessary previous nodes: this technique is performed by traversing from the root node of the graph until it finds the first predicate or update operation on a table or a field. Then nodes which are prior to the table or the table of the field can be eliminated from the graph.

2. Eliminate join of any two contiguous tables: Define: $\mathrm{T} 1$ and $\mathrm{T} 2$ are two contiguous tables starting from the root of the graph. PK stands for primary key and FK stands for foreign key.

On the graph, if $\mathrm{T} 1$ consists of only one field which is $\mathrm{PK} / \mathrm{FK}$ linking to $\mathrm{FK} / \mathrm{PK}$ of $\mathrm{T} 2$ and $\mathrm{P}$ is a predicate on $\mathrm{PK} / \mathrm{FK}$ of $\mathrm{T} 1$, then $\mathrm{P}$ can be moved to FK/PK of T2 and T1 and its $\mathrm{PK} / \mathrm{FK}$ can be eliminated from the graph.

3. Eliminate join of any three contiguous tables:

Define: T1, T2 and T3 are three contiguous tables starting from the root of the graph.

On the graph, if T2 consists of only one field which is $\mathrm{PK} / \mathrm{FK}$ linking to $\mathrm{FK} / \mathrm{PK}$ of $\mathrm{T} 1$ and $\mathrm{FK} / \mathrm{PK}$ of T3 then T1 and T3 can be joined together directly and $\mathrm{T} 2$ and its $\mathrm{PK} / \mathrm{FK}$ can be eliminated from the graph. If there is a predicate on $\mathrm{PK} / \mathrm{FK}$ of $\mathrm{T} 2$ then the predicate will be moved to FK/PK of T3.

Note: If a graph is already in optimized form, the optimization will not be applied.

\subsection{Steps for Translating XML Update Language}

The steps for translating XML update language into SQL are given below:

1. Rewrite the update command to SQL functions according to the rewriting rules.

2. Create a graph whose paths correspond to paths in the functions.

3. Map the graph into the database schema graph to identify which node is a table or field.

4. Add key fields (PK and FK) which are used to join tables. However, in the case of recursion on the path of the command (keys of elements referring back to ancestors in the path of the command) key fields will not be added. Then add the join symbols by using the capital L followed by numbers (L1, L2, $\ldots, \mathrm{Ln})$ to indicate which pair of the keys is used to join the tables.

5. Map the functions to the graph If an insert function is performed on a node converted to the primary key of a table, this insert function must be copied to the foreign key of child-tables to maintain parent-child relationships.

If a delete or insert function is performed on nodes converted to fields, without a delete or insert function on an ancestor-node converted to a table, the function will be converted to an update function.

If an insert or delete function is performed on a node converted to a table, this indicates that the function will insert or delete a row of the table. In this case the function will not be converted to an update function. If an update, where or group_by function is performed on a node converted to a table, the function will be pushed down to the appropriate primary key of the table.

6. In the case that there is more than one update function on different tables, the graph will be split into sub-graphs. The number of sub-graphs is equal to the number of update operations performed on the different tables having different funcNo. 
7. For each sub-graph, some join symbols can be eliminated when

- only one table is involved in the updating.

- an update statement and all of its where clauses are in the same table.

- a select statement and all of its where clauses are in the same table.

8. Optimize each (sub-) graph according to optimization rules.

9. Generate SQL commands or update/delete join commands from each (sub-)graph. The insert functions, omitting the second parameter (value |:funcNo) and the bindF/bindL functions, will be ignored in generating the commands.

10. If the generated commands are in the form of update/delete join commands, the commands are rewritten according to the SQL rewriting rules.

\subsection{An example of Translating XML Update Language into SQL}

For $\$ p$ in $\operatorname{doc}$ ("Library.xml")/Publication, $\$ \mathrm{a}$ in $\$ \mathrm{p} /$ Author

Where $\$ \mathrm{p} /$ Title $=$ "Java"

Delete \$p/PubType, Delete \$a

Where \$a/Name = 'John S.'

1. Parse the command and rewrite it to SQL functions as follows

bindF (/Publication, \$p, 0)

bindF ( $\$ \mathrm{p} /$ Author, $\$ \mathrm{a}, 0)$

where $(\$ \mathrm{p} /$ Title, $=$, 'Java', 0)

$\operatorname{delete}(\$ \mathrm{p} /$ PubType, 1) delete $(\$ \mathrm{a}, 2)$

where $(\$ a /$ Name, $=$, 'JohnS.', 2)

2. Create a graph whose nodes correspond to paths in the functions, map the graph to the database schema graph, add key fields (PK

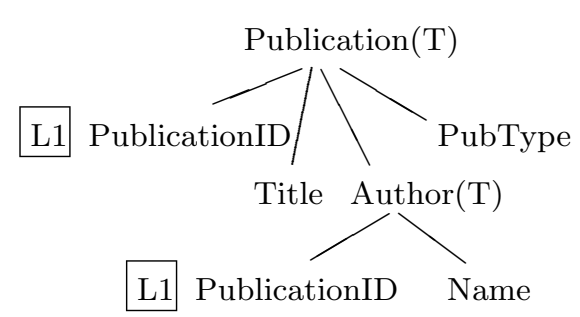

(a) Graph after mapping database schema graph

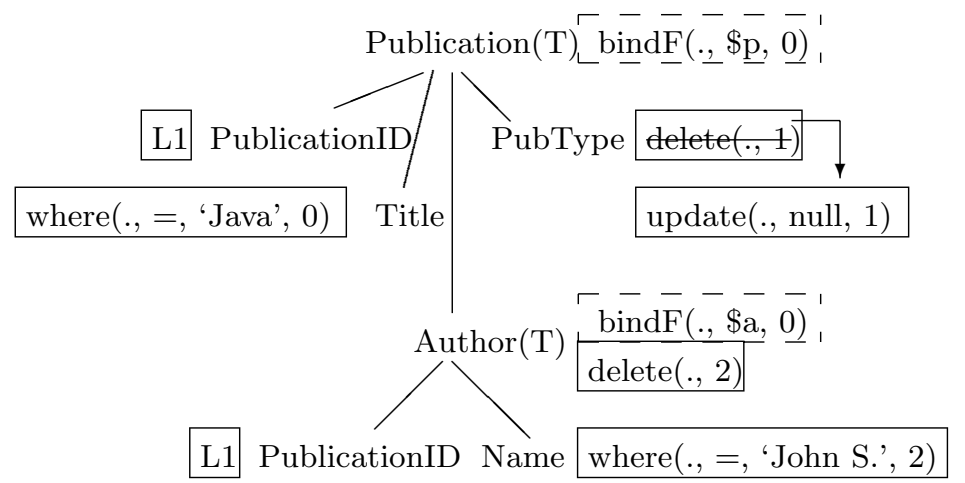

(b) Graph after mapping SQL function and after delete function is changed to update function

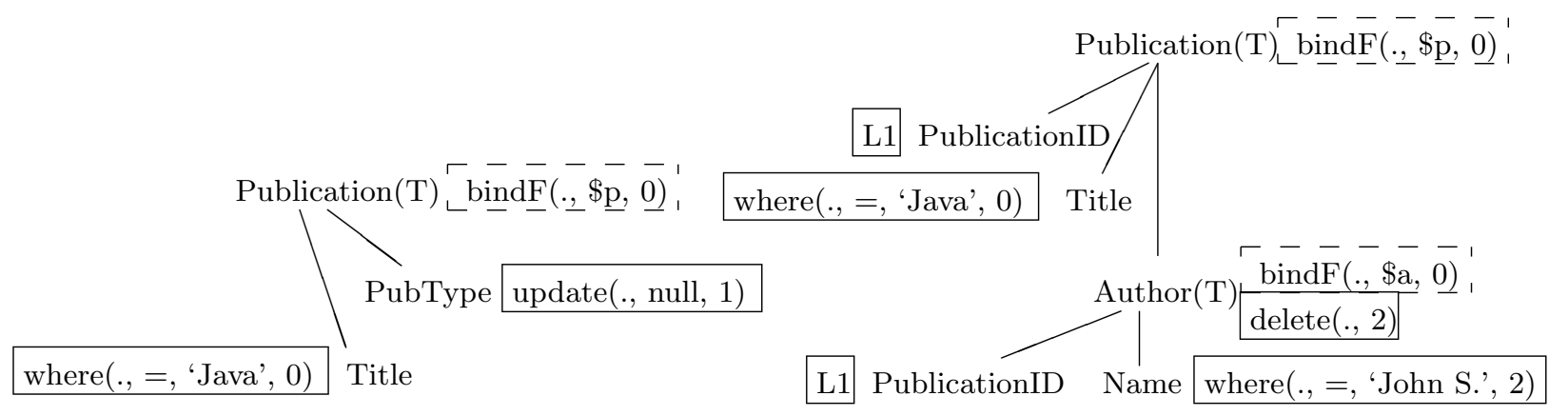

(c) Two sub-graphs of updating Publication table and Author table respectively 
and FK) which are used to join tables and finally add the join symbols to indicate which pair of keys is used to perform the join between tables. The result is shown in Figure 6(a).

3. Map the SQL functions to the graph. Since the delete function is performed on the node 'PubType' converted to a field and there is no delete function on the ancestor of this node converted to a table, then the function is changed to an update function The result is shown in Figure 6(b).

4. Split the graph into sub-graphs. There are two updated target tables: Publication and Author. Therefore the graph is split into two sub-graphs as shown in Figure 6(c).

5. Each sub-graph is already in optimized form. So commands can be generated from each sub-graph as follows: For the first subgraph, only one table is involved in the update; thus, there is no join in the update operation so the SQL command is generated as follows:

Update Publication P

Set P.PubType $=$ null

Where P.Title = 'Java';

For the second sub-graph, a delete join command is generated as follows:

Delete Author A

From A, Publication P

Where P.Title = 'Java'

And P.PublicationID = A.PublicationID

And A.Name = 'John S.';

The delete join command is rewritten as an SQL command with a sub-query by using the SQL rewriting rules as follows:

Delete Author A

Where A.AuthorID in

(Select A.AuthorID

From Author A, Publication P

Where P.Title = 'Java'

And P.PublicationID = A.PublicationD

And A.Name = 'John S.');

\section{Translating the Recursive Function into PL/SQL}

The recursive function is processed in the manner of loop processing, whereas a loop structure cannot be translated into pure SQL commands. Thus a possible way is translating the function into some $\mathrm{SQL}$ forms such as persistent stored modules (standard SQL) or PL/SQL (Oracle). In our research, the recursive function will be translated into PL/SQL because it is a very well designed tool in Oracle.

In our translation, we apply the concept of variables to the concept of tables since only the tables can be directly manipulated by SQL commands. In this section, firstly, the mechanism for passing a variable's value is described. Secondly, the rewriting rules for translating the recursive function are proposed. Thirdly, steps translating the recursive function into $\mathrm{PL} / \mathrm{SQL}$ are presented and finally an example is expressed.

\subsection{The Mechanism for Passing a Variable's Value}

The mechanism for passing a variable's value is applied to selecting and inserting data from/into tables as follows.

1. The concept of passing a value of a variable to another variable is applied to the concept of selecting data from a table and then inserting this data into another table.

2. The notion of passing a variable's value is that the old value in a variable will be overwritten with the new value passed by another variable. To use tables instead of variables means that before inserting the data into a table, the old data in the table must be deleted.

\subsection{Rewriting Rules for Translating the Recursive Function}

The clauses of the XML update command will be rewritten as SQL functions or SQL-syntax commands subject to the following rules.

1. In the rewriting rules for $\mathrm{FLW}(\mathrm{R}|\mathrm{I}| \mathrm{D})$ expression, the Where|Replace|Insert|Delete clause is rewritten as an SQL function as mentioned earlier, whereas the rule of For|Let clause is changed. Instead of binding variables to nodes in XPath, the meaning of operation in For|Let clause is interpreted as the meaning of the operation in SQL because the number for calling the function is dynamic 
depending on the result derived from the previous loop processing. Thus the number of binding variables cannot be determined in advance and hence the rule is as follows.

For \$var in XPathExp |

Let \$var := XPathExp

is rewritten as:

Insert into \$var select(XPathExp, funcNo)

2. The variable which passes value in the calling function is called 'argument' while the variable which receives the value from the argument is called 'parameter'. Thus

In function call, passing \$argument to \$parameter

is rewritten as:

Insert into \$parameter Select * from \$argument

3. Replace the variables (parameter, argument) in SQL functions or SQL-syntax commands derived from rules 1 and 2 with their corresponding tables and/or elements. The variables are categorized into two types: variables which are not a part of XPathExp (independent variables) and variables which are a part of XPathExp. Thus the rules for replacing variables are as follows:

The independent variables will be replaced with tables. Here we define that the argument will be superseded by table 'Array', whereas the parameter will be substituted with table 'ProcessingArr'; thus

If $\$$ var is argument then

\$var

is rewritten as: table 'Array'

ElseIf \$var is parameter then

\$var

EndIf

table 'ProcessingArr'

is rewritten as:

\section{The variable which is a part of XPathExp} cannot be replaced with tables directly. There are two cases for these variables: variables in a where function and variables in other SQL functions which are not where functions. In both cases, the variables will be replaced with their corresponding elements. In the case of the function which is not a where function, besides replacing the variable with its corresponding element, the following condition must be specified: the value in the element must be the same as the value held in the variable. The variable must be replaced with table Array/ProcessingArr depending on whether the variable is an argument or a parameter; thus it means that the value in the element must be the same as the data kept in the table. Therefore, the rules are as follows.

Suppose that E1 is the element corresponding to the variable \$var; ergo

where (\$var/XPath, funcNo) is rewritten as: where(E1/XPath, funcNo)

Suppose that E1 is the element corresponding to the variable \$var and SQLFunct is any SQL function which is not the function 'where'; ergo

SQLFunct (\$var/XPath, funcNo)

is rewritten as:

SQLFunct(E1/XPath, funcNo)

where (E1, in, , funcNo)

( select $*$ from Array/ProcessingArr)

\subsection{Steps for Translating the Recursive Function into a PL/SQL Command}

1. Rewrite each clause of the update command until the first calling function in the body of function is found by using the rules $1-2$. The first calling function will not be rewritten in this step.

2. Create a loop structure when the first calling function in the body of the function is found. In the loop, the first calling function and each clause in the body of function is rewritten by using the rules $1-2$. The second calling function will not be rewritten.

3. Replace the variables in SQL functions and SQL-syntax commands derived from steps $1-2$ by using rule 3 .

4. Follow the concept of passing a variable's value; therefore before inserting data into tables, the old value in such tables must be deleted; thereby each clause for insertion of data is preceded by a clause for deleting old data in the table.

5. Translate SQL functions embedded in PL/ SQL into SQL commands by using graph mapping, as mentioned in translating XML update language into SQL. 


\subsection{An Example of Translating the Recursive Function into PL/SQL}

In this example we want to update the year of both direct and indirect references of the publication whose title is 'XQuery'. The recursive function and the command calling the function are as follows. (This function follows the syntax of XQuery)

1 define function allRef $\left(\$\right.$ pub as element ()$\left.^{*}\right)$ 2\{

\section{For $\$ r$ in}

\$pub/Reference/@RefPub->Publication

4 Replace \$rp/Year with < Year >2004</Year>

5 allRef $(\$ r p)$

$6\}$

7 For $\$ p$ in doc("Library.xml")/Publication

8 Where $\$ \mathrm{p} /$ Title $=$ "XQuery"

9 allRef $(\$ \mathrm{p})$

The process starts from the clause in line 7 . Then it is translated into PL/SQL as follows.

\begin{tabular}{|c|c|}
\hline Clauses in the update command & Result of rewriting \\
\hline $\begin{array}{l}7 \text { For } \$ p \text { in doc("Library.xml")/Publication } \\
8 \text { Where } \$ \text { p/Title = "XQuery" }\end{array}$ & $\begin{array}{l}\text { Insert into } \$ \mathrm{p} \\
\text { select }(/ \text { Publication, } 0) \\
\text { where }(\$ \mathrm{p} / \text { Title, }=, \text { 'XQuery', 0); }\end{array}$ \\
\hline $\begin{array}{l}9 \text { allRef }(\$ \mathrm{p}) \\
1 \text { define function allRef }\left(\$ p u b \text { as element }()^{*}\right)\end{array}$ & $\begin{array}{l}\text { Insert into } \$ \mathrm{pub} \\
\text { Select } * \text { from } \$ \mathrm{p} ;\end{array}$ \\
\hline 3 For $\$ r p$ in $\$$ pub/Reference/@RefPub->Publication & $\begin{array}{l}\text { Insert into \$rp } \\
\text { select(\$pub/Reference/@RefPub->Publication, 1); }\end{array}$ \\
\hline 4 Replace $\$ \mathrm{rp} /$ Year with $<$ Year $>2004</$ Year $>$ & update (\$rp/Year, '2004', 2); \\
\hline
\end{tabular}

Fig. 7. Result of rewriting clauses in the update command.

\begin{tabular}{|l|l|}
\hline \multicolumn{1}{|c|}{ Clauses in loop } & \multicolumn{1}{c|}{ Result of rewriting } \\
\hline $\begin{array}{l}5 \text { allRef }(\$ \mathrm{rp}) \\
1 \text { define function allRef }\left(\$ \mathrm{pub} \text { as element }()^{*}\right)\end{array}$ & $\begin{array}{l}\text { Insert into \$pub } \\
\text { select * from \$rp; }\end{array}$ \\
\hline 3 For \$rp in \$pub/Reference/@RefPub->Publication & $\begin{array}{l}\text { Insert into \$rp } \\
\text { select }(\$ p u b / R e f e n c e / @ R e f P u b->\text { Publication, 3); }\end{array}$ \\
\hline 4 Replace \$rp/Year with $<$ Year $>2004</$ Year $>$ & update $(\$ r p /$ Year, '2004', 4); \\
\hline
\end{tabular}

Fig. 8. Result of rewriting clauses in the body of the function which are processed in loop.

\begin{tabular}{|c|c|}
\hline Clauses in the update command & Result of replacing variables \\
\hline $\begin{array}{l}\text { Insert into } \$ \mathrm{p} \\
\text { select }(/ \text { Publication, 0) } \\
\text { where }(\$ \mathrm{p} / \text { Title, =, 'XQuery', 0); }\end{array}$ & $\begin{array}{l}\text { Insert into Array } \\
\text { select(/Publication, 0) } \\
\text { where(/Publication/Title, =, 'XQuery', 0); }\end{array}$ \\
\hline $\begin{array}{l}\text { Insert into } \$ \mathrm{pub} \\
\text { Select * from } \$ \mathrm{p} ;\end{array}$ & $\begin{array}{l}\text { Insert into ProcessingArr } \\
\text { Select * from Array; }\end{array}$ \\
\hline $\begin{array}{l}\text { Insert into \$rp } \\
\text { select(\$pub/Reference/@RefPub->Publication, 1); }\end{array}$ & $\begin{array}{l}\text { Insert into Array } \\
\text { select(/Publication/Reference/@RefPub->Publication, 1) } \\
\text { where(/Publication, in, } 1) \\
\text { (Select* from ProcessingArr); }\end{array}$ \\
\hline update(\$rp/Year, '2004', 2); & $\begin{array}{l}\text { update(/Publication/Year, '2004', 2) } \\
\text { where(/Publication, in, , 2) } \\
\text { (Select * from Array); }\end{array}$ \\
\hline
\end{tabular}

Fig. 9. Result of replacing variables in clauses which are outside loop. 


\begin{tabular}{|c|c|}
\hline Clauses in loop & Result of replacing variables \\
\hline $\begin{array}{l}\text { Insert into } \$ \text { pub } \\
\text { select } * \text { from } \$ r p\end{array}$ & $\begin{array}{l}\text { Insert into ProcessingArr } \\
\text { Select }{ }^{*} \text { from Array; }\end{array}$ \\
\hline $\begin{array}{l}\text { Insert into \$rp } \\
\text { select }(\$ p u b / \text { Reference/@RefPub->Publication, 3); }\end{array}$ & $\begin{array}{l}\text { Insert into Array } \\
\text { select(/Publication/Reference/@RefPub->Publication, 3) } \\
\text { where(/Publication, in, ,3) } \\
\text { (Select * from ProcessingArr); }\end{array}$ \\
\hline update $(\$ r p / Y e a r, ~ ' 2004 ', 4)$; & $\begin{array}{l}\text { update(/Publication, '2004', 4) } \\
\text { where(/Publication, in, ,4) } \\
\text { (Select * from Array); }\end{array}$ \\
\hline
\end{tabular}

Fig. 10. Result of replacing variables in clauses which are inside loop.

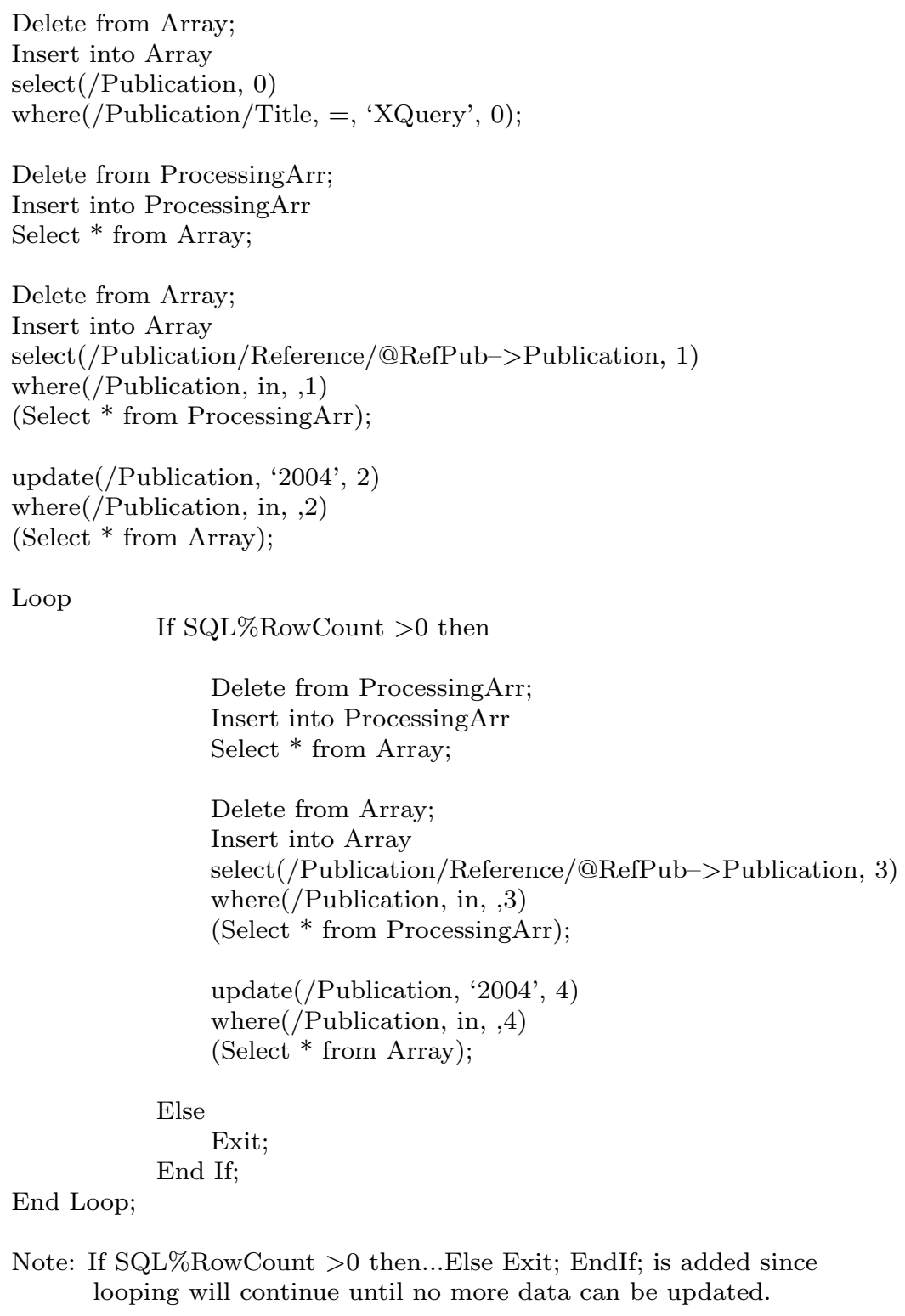

Note: If SQL\%RowCount $>0$ then...Else Exit; EndIf; is added since looping will continue until no more data can be updated.

Fig. 11. Adding a delete clause before each insertion of data into tables. 
1. Rewrite each clause until the first calling function in the body of function is found. The clauses in the update command and the result of rewriting these clauses by using rewriting rules 1-2 are shown in Figure 7.

2. Create loop structure (Loop....EndLoop) when the first calling function in the body of function is found. Clauses in the loop which are rewritten by using rewriting rules 1-2 are shown in Figure 8.

3. Replace the variables by using rule 3 . The results after replacing variables are shown in Figures 9 and 10.

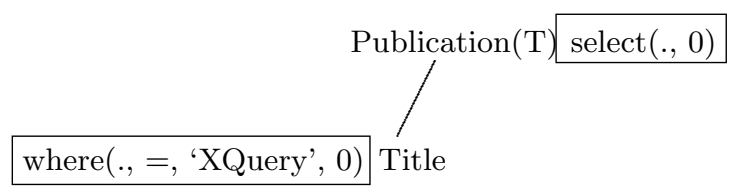

(a) Graph of SQL function in group 0

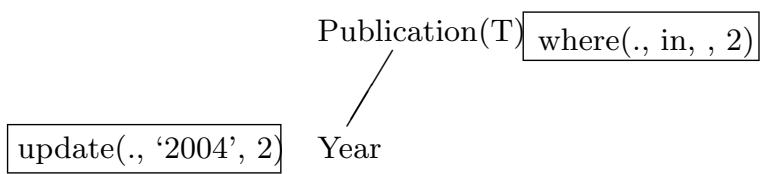

(c) Graph of SQL function in group 2
4. Follow the concept of passing a variable's value; thus each clause for insertion of data is preceded by a clause for deleting old data in the table. The result which is a PL/SQL command is shown in Figure 11.

5. Translate SQL functions embedded in PL/ SQL into SQL commands. From Figure 11, there are 5 groups of SQL functions identified by the function number 0-4. However SQL functions in group 1 are the same as the ones in group 3 and SQL functions in group 2 are the same as the ones in group 4. Thus we will only show translating SQL functions

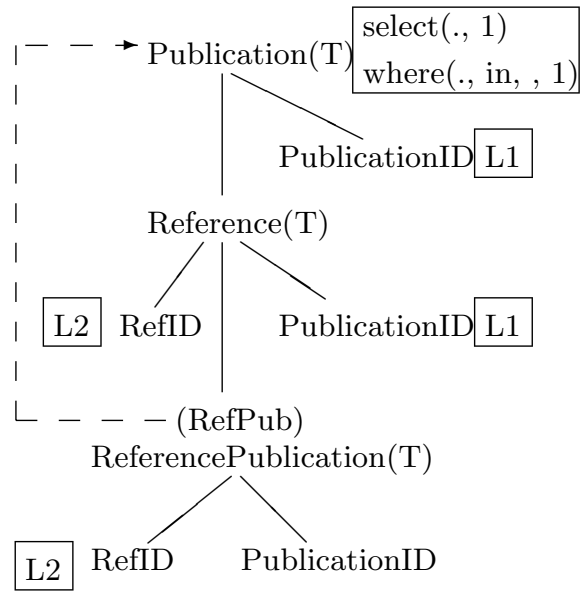

(b) Graph of SQL function in group 1

Fig. 12.

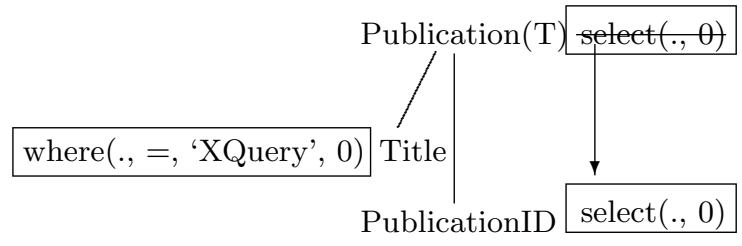

(a) Graph after pushing select function in group 0

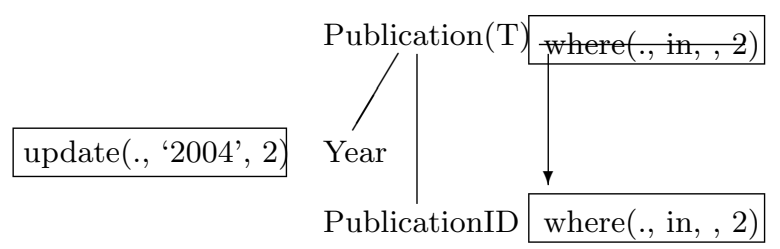

(c) Graph after pushing where function in group 2

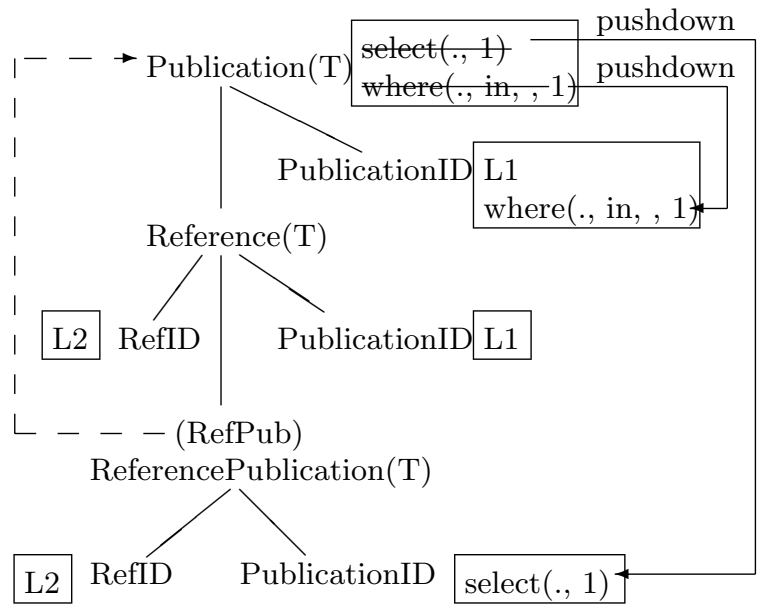

(b) Graph after pushing select and where functions in group 1

Fig. 13. 


\begin{tabular}{|c|c|}
\hline SQL functions & SQL commands \\
\hline $\begin{array}{l}\text { select }(/ \text { Publication, } 0) \\
\text { where(/Publication/Title, =, 'XQuery', 0); }\end{array}$ & $\begin{array}{l}\text { Select P.PublicationID } \\
\text { From Publication P } \\
\text { Where P.Title = 'XQuery'; }\end{array}$ \\
\hline $\begin{array}{l}\text { select }(/ \text { Publication/Reference/@RefPub->Publication, 1) } \\
\text { where(/Publication, in, , 1) }\end{array}$ & $\begin{array}{l}\text { Select RP.PublicationID } \\
\text { From Publication P, Reference R, ReferencePublication RP } \\
\text { Where RP.RefID = R.RefID } \\
\text { And R.PublicationID = P.PublicationID } \\
\text { And P.PublicationID in }\end{array}$ \\
\hline $\begin{array}{l}\text { update(/Publication/Year, '2004', 2) } \\
\text { where(/Publication, in, , 2) }\end{array}$ & $\begin{array}{l}\text { Update Publication P } \\
\text { Set P.Year = '2004' } \\
\text { Where P.PublicationID in }\end{array}$ \\
\hline $\begin{array}{l}\text { select(/Publication/Reference/@RefPub->Publication, 3) } \\
\text { where(/Publication, in, , 3) }\end{array}$ & $\begin{array}{l}\text { Select RP.PublicationID } \\
\text { From Publication P, Reference R, ReferencePublication RP } \\
\text { Where RP.RefID = R.RefID } \\
\text { And R.PublicationID = P.PublicationID } \\
\text { And P.PublicationID in }\end{array}$ \\
\hline $\begin{array}{l}\text { update(/Publication/Year, '2004', 4) } \\
\text { where(/Publication, in, , 4) }\end{array}$ & $\begin{array}{l}\text { Update Publication P } \\
\text { Set P.Year = '2004' } \\
\text { Where P.PublicationID in }\end{array}$ \\
\hline
\end{tabular}

Fig. 14. SQL commands generated from the graphs.

in groups 0,1 and 2. SQL commands will be generated from each group of SQL functions independently as follows:

(a) Graphs of SQL functions in groups 0,1 and 2 are created according to paths in SQL functions.

(b)The graphs are mapped to the database schema graph, join keys and join symbols are added to the graphs and finally each group of SQL functions is mapped to its corresponding graph. The results of mapping SQL functions in groups 0,1 and 2 are shown in Figures 12(a), 12(b) and $12(\mathrm{c})$ respectively.

(c) The select function in group 1 acting on Publication element is pushed down to the field linking to this element since this function is performed on the node which is on the recursive path. The select function in group 0 , where function in group 1 and where function in group 2 acting on Publication element converted to table are pushed down to the primary key of this table. The results of pushing SQL functions in groups 0,1 and 2 are shown in Figures 13(a), 13(b) and 13(c) respectively.
(d)SQL commands generated from the graphs are shown in Figure 14. The SQL functions in groups 3 and 4 are the same as the ones in groups 1 and 2 respectively; hence the generated SQL commands from the functions in groups 3 and 4 will be the same as the generated SQL commands from the functions in groups 1 and 2 respectively.

(e) SQL functions in the PL/SQL command are replaced with the SQL commands generated from the graphs. The result is shown in Figure 15.

\section{Conclusion and Further Work}

Our work translates five important features of the XML update language inherited from XQuery into SQL: FLW $(\mathrm{R}|\mathrm{I}| \mathrm{D})$, conditional expression, quantifier, aggregate functions and (nonrecursive) user-defined function. Four techniques are used: rewriting rules, graph mapping, optimization and update/delete join commands. The recursive function is translated into PL/SQL by applying the concept of variable to the notion of table and then using graph mapping technique to generate SQL commands 


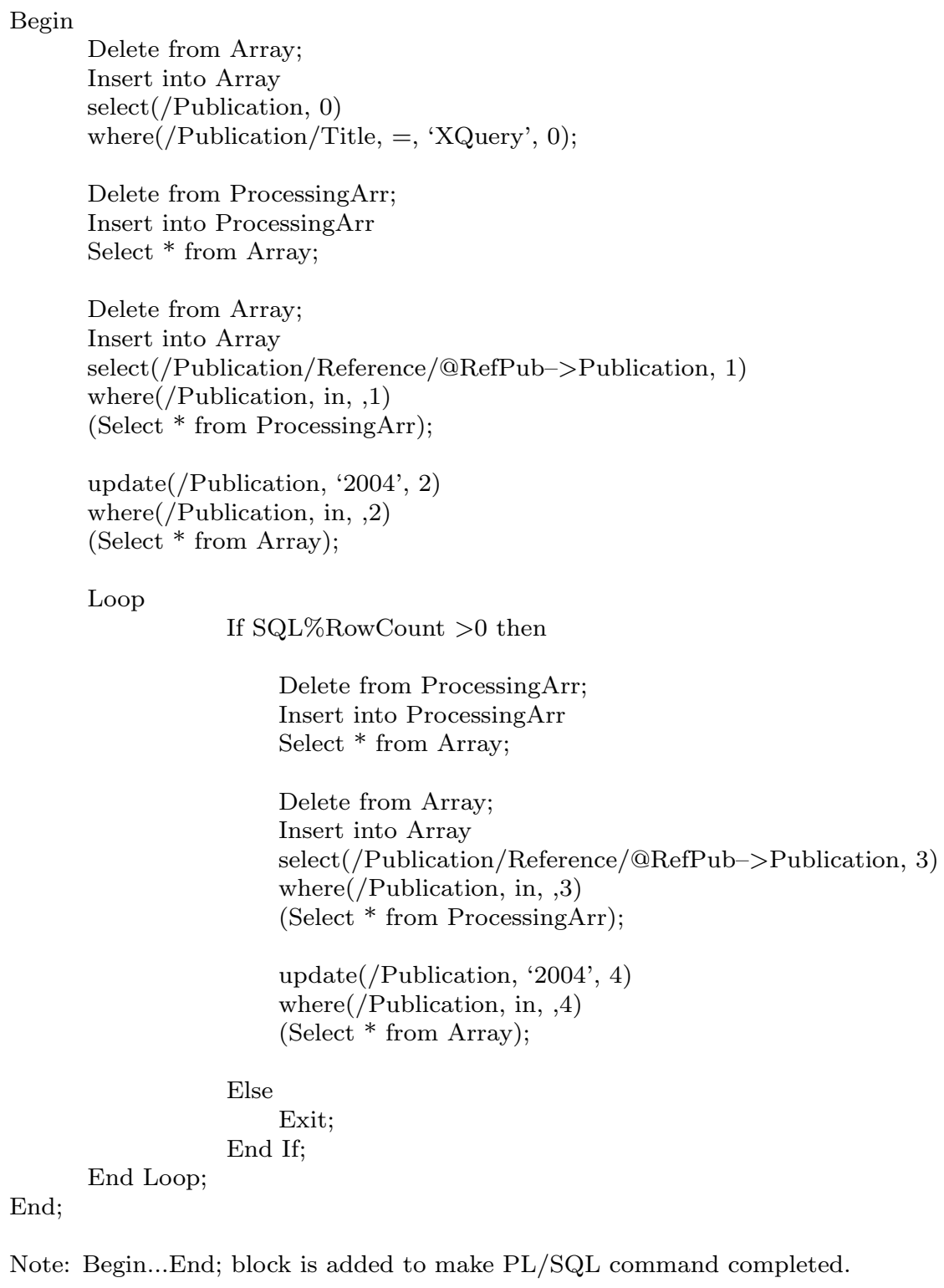

Note: Begin...End; block is added to make PL/SQL command completed.

Fig. 15. PL/SQL command after replacing SQL functions.

from SQL functions. One major benefit of updating XML documents through the database is that presevering constraints can be pushed to the database engine. Our translating approach can apply to updating other (object) relational databases whose schemas are derived from mapping XML documents by the shredding approach. Examples of translating XML update language into SQL to update objectrelational database are presented on our website [2].

In our further work, we will propose how to han- dle the order of elements in XML documents when elements are inserted or deleted and we will present a mechanism for propagating the change in the database to the XML documents.

\section{References}

[1] S. Abiteboul, D. QuAss, J. McHuge, J. Widom AND J. L. WINER, The Lorel query language for semistructured data. Proceedings of International Journal on Digital Libraries, (1997), pp. 68-88. 
[2] P. Amornsinlaphachai, N. Rossiter AND A. Ali, Translating XML update language into SQL based upon object relational database. 2005: http://computing.unn. ac.uk/pgrs/cgpa2/.

[3] S. Ceri, S. Comai, E. DAmiani, P. Fraternali, S. PARABOSCHI AND L. TANCA, XML-GL: a Graphical Language for Querying and Restructuring WWW Data. Computer Networks: The International Journal of Computer and Telecommunications Networking, 31 (1999), pp. 1171-1187.

[4] D. CHAMBERLIN, XQuery from experts: A Guide to the W3C XML Query Language. Addison-Wesley, (2003).

[5] A. Deutsch, M. Fernandez, D. Florescu, A. LEVY AND D. SUCIU, A query language for XML. Proceedings of the 8th International World Wide Web Conference (WWW8), Toronto. Canada, (1999).

[6] A. Deutsch, M. Fernandez And D. Suciu, Storing Semistructured Data with STORED. SIGMOD Conference, Pennsylvania, United States, (1999), pp. 431-442.

[7] M. Fernandez, Y. KadiysKa, D. SUCIU, A. MoRISHIMA AND W. TAN, SILKROUTE, A Framework for Publishing Relational Data in XML. ACM Transactions on Database Systems (2002), pp. 1-55.

[8] M. Fernandez, A. Morishima And D. Suciu, Efficient Evaluation of XML Middle-ware Queries. ACM SIGMOD, Santa Barbara, California, USA, (2001).

[9] D. Florescu, I. Manolescu and D. Kossmann, Answering XML Queries over Heterogeneous Data Sources. Proceedings of the 27th VLDB Conference, Roma, Italy, (2001).

[10] J. Fong AND T. DILLON, Towards Query Translation From XQL to SQL. Proceedings of the 9th IFIP 2.6 working conference on database semantics (DS9), Hong Kong, (2001), pp. 113-129.

[11] S. JAIN, R. MAHAJAN AND D. Suciu, Translating XSLT Programs to Efficient SQL Queries. Proceedings of the eleventh international conference on World Wide, ACM Press New York, NY, USA, Honolulu, Hawaii, USA, (2002), pp. 616-626.

[12] E. C. JENSEN, S. M. Beitzel And D. A. GRossman, Using a Relational Database Management System to Implement XML-QL. Proceedings of the 17th International Conference on Advanced Science and Technology (ICAST'2001), Chicago, (2001).

[13] B. KANE, Consistently Updating XML Documents using Incremental Constraint Check with XQueries. Worchester Polytechnic Institute, (2003).

[14] L. KHAN AND Y. RAO, A Performance Evaluation of Storing XML Data in Relational Database Management Systems. ACM (2001).

[15] M. KLETTKE AND H. MEYER, Managing XML Documents in object-relational databases. Computer Science Department, University of Rostock, Rostock, Germany, (1999).
[16] R. Krishnamurthy, V. T. Chakaravarthy, R. KAUSHIK AND J. F. NAUGHTON, Recursive XML Schema, Recursive XML Queries, and Relational Storage: XML-toSQL Query Translation. ICDE 2004 (2004).

[17] D. LEE AND W. W. CHU, Constraints-preserving Transformation from XML Document Type Definition to Relational Schema. 19th International Conference on Conceptual Modeling, Salt Lake City, Utah, USA, (2000), pp. 323-338.

[18] D. LEE AND W. W. CHU, CPI: ConstraintsPreserving Inlining Algorithm for Mapping XML DTD to Relational Schema. Data \& Knowledge Engineering, 39 (2001), pp. 3-25.

[19] M. LIU, L. LU AND G. WANG, A Declarative XMLRL Update Language. Proceedings of 22nd International Conference on Conceptual Modeling (ER 2003), Springer-Verlag, Chicago, Illinois, USA, (2003), pp. 506-519.

[20] I. Manolescu, D. Florescu AND D. Kossmann, Pushing XML Queries inside Relational Databases. INRIA Technical Report No. 4112, (2001).

[21] J. McGoveran, P. Bothner, K. Cagel, J. LinN AND V. NAGARAJAN, XQuery Kick Start. Sams Publishing, (2003).

[22] Y. Mo AND L. T. WANG, Storing and Maintaining Semistructured Data Efficiently in an ObjectRelational Database. The Third International Conference on Web Information Systems Engineering, Singapore, (2002), pp. 247-256.

[23] J. RoBIE, The Design of XQL. 1999: http : //www . ibiblio.org/xql/xql-design.html.

[24] T. SCHLIEDER, Querying and ranking XML documents. Journal of the American Society for Information Science and Technology, 53 (2002), pp. 489-503.

[25] A. Schmidt, M. Kersten, M. Windhouwer AND F. WASS, Efficient Relational Storage and Retrieval of XML documents. International Workshop on the Web and Databases, Dallas, TX, USA, (2000), pp. 47-52.

[26] Shamkante B. Navathe, A Proposal for an XML Data Definition and Manipulation Language. VLDB Conference, Hong Kong China, (2002).

[27] J. Shanmugasundaram, J. KIERnAN, E. SHEKITA, C. FAN AND J. FUNDERBURK, Querying XML Views of Relational Data. Proceedings of the 27th VLDB Conference, Roma. Italy, (2001).

[28] J. Shanmugasundaram, K. Tufte, G. He, C. ZhANG, D. J. DEWITT AND J. F. NAUGHTON, Relational Databases for Querying XML Documents: Limitations and Opportunities. Proceedings of the 25th VLDB Conference, Edinburgh, Scotland, (1999), pp. 302-314.

[29] T. SHIMURA, M. YoshiKaWA AND S. UEMURA, Storage and Retrieval of XML Documents using Object-Relational Databases. IPSJ Transactions on Databases Abstract, 40 (2001). 
[30] I. Tatarinov, Z. IVeS, A. Y. Halevy And D. S. WELD, Updating XML. Proceedings of 2001 SIGMOD Conference, Santa Barbara, CA, USA., (2001), pp. 413-424.

[31] I. VARLAMIS AND M. VAZIRGIANNIS, Bridging XML-Schema and relational databases. A system for generating and manipulating relational databases using valid documents. ACM Symposium on Document Engineering (2001), pp. 105-114.

[32] W3C: XQuery 1.0 and XPath 2.0 Data Model. W3C working draft. 2003: http: //www.w3.org/ TR/query-dat amodel.

[33] W3C: XQuery 1.0: An XML Query Language. 2003: http://www.w3c.org/TR/xquery.

[34] XML:DB working group: XUpdate. 2002. http: //www.xmldb.org/xupdate/xupdatewd.html: http://www.xmldb.org/xupdate /xupdate-wd.html.

[35] M. YoshiKawa, T. Amagasa, T. SHIMURA AND S. UEMURA, XREL: A Path-Based Approach to Storage and Retrieval of XML documents using Relational Databases. ACM Transactions on Internet Technology, 1 (2001).

Received: October, 2004 Revised: July, 2005 Accepted: September, 2005

Contact address:

Pensri Amornsinlaphachai School of Computing, Engineering \& Information Sciences Northumbria University

Pandon Building (Room 113), Camden Street, Newcastle upon Tyne, NE2 1XE, UK e-mail: pensri.amornsinlaphachai@unn.ac.uk

PENSRI AMORNSINLAPHACHAI is a Ph.D. student at School of Computing, Engineering \& Information Sciences, Northumbria University, Newcastle, UK. She received her MSc. with Distinction in 2001 and Sun Certified Programmer For THE JAVA 2 in 2002.

DR NICK ROSSITER is a reader at School of Computing, Engineering and Information Sciences, Northumbria University, Newcastle, UK. He is interested in interoperability of information systems.

DR M. AKHTAR ALI is a senior lecturer at School of Computing, Engineering and Information Sciences, Northumbria University, Newcastle, UK. He received his Ph.D. in 2003 from Manchester University. 